\title{
Dimension Increase and Splitting for Poincaré-Dulac Normal Forms
}

\author{
Giuseppe GAETA† and Sebastian WALCHER ${ }^{\ddagger}$ \\ † Dipartimento di Matematica, Università di Milano \\ I-20133 Milano, Italy \\ E-mail: gaeta@mat.unimi.it \\ ¥ Lehrstuhl A für Mathematik, RWTH Aachen \\ D-52056 Aachen, Germany \\ E-mail: walcher@mathA.rwth-aachen.de
}

This article is part of the special issue published in honour of Francesco Calogero on the occasion of his 70th birthday

\begin{abstract}
Integration of nonlinear dynamical systems is usually seen as associated to a symmetry reduction, e.g. via momentum map. In Lax integrable systems, as pointed out by Kazhdan, Kostant and Sternberg in discussing the Calogero system, one proceeds in the opposite way, enlarging the nonlinear system to a system of greater dimension. We discuss how this approach is also fruitful in studying non integrable systems, focusing on systems in normal form.
\end{abstract}

\section{Introduction. Dimension increase and splitting}

Finite dimensional integrable systems have been a rarity for a very long time; up to few decades ago, they could be easily enumerated: harmonic oscillators, the Kepler system, a few spinning tops.

Apart from harmonic oscillators - which give linear equations and are the prototype of integrability - these are integrated by exploiting their symmetries and the associated integrals of motion to reduce the problem to a lower dimensional one; thus, we have symmetry reduction.

The situation changed radically in the seventies, when entire classes of new integrable systems appeared, integrable by the Lax pair construction [14]. Among these a prominent role - also due to priority - is taken by the Calogero system [3]. In this case, one has an arbitrary number $N$ of points on the line, interacting via a certain pair potential; thus the system is described by a natural hamiltonian $H=(1 / 2) \sum_{i}\left(\dot{x}_{i}\right)^{2}+V(x)$. Calogero showed that there is an invertible map from $\mathbf{R}^{N}$ to $G L(N)$, mapping the vector $x$ to a matrix $L(x)$, such that the evolution of $x(t)$ results in an evolution of $L(t):=L[x(t)]$ governed by Lax equations; thus one integrates $L(t)$ and obtains $x(t)$. 
As pointed out by Kazhdan, Kostant and Sternberg [13], this also initiated a new way of integrating systems (which they studied geometrically): indeed, rather than trying to reduce the dimension of the system, Calogero passed to consider a system of higher dimension $^{1}$. In this note we want to show that this approach is fruitful also beyond the realm of integrable hamiltonian systems.

The key observation made by Kazhdan, Kostant and Sternberg [13], see also [15, 16], in the context of Hamiltonian systems is that given a differential equation $\Delta$ in dimension $n$, in some cases it may be helpful to increase its dimension: this is in particular the case when it is possible to describe $\Delta$ as originating from either the symmetry reduction or the projection to an $n$-dimensional manifold of a simpler (e.g. linear) equations $\Delta^{*}$ in dimension $n+m$.

It was remarked in [10] that this approach does apply also to non-hamiltonian systems, and in particular to systems in Poincaré-Dulac normal form [1, 5, 20] (a remark to this effect was already contained in [20]). Essentially, a system in normal form with only a finite number of resonances can always be mapped to a linear system, and is equivalent to the latter on a certain invariant manifold. As observed there, such a procedure is geometrically interesting, but equivalent to a classical technique already known to Dulac [6], and abscribed by him to Horn and Lyapounov. In more general terms, i.e. outside the scope of normal forms theory, this corresponds to the situation of a finite dimensional centralizer for a certain algebra associated to the vector field [11].

In the present note, we show how "dimension increasing" nicely combines with a "splitting" (in a sense to be described below) approach [8] in the case of an infinite number of resonances $^{2}$; this case cannot be tackled by the Horn-Lyapounov-Dulac approach.

Roughly speaking, the procedure presented in this note combines the two approaches mentioned above, i.e. describing a nonlinear system as projection of a linear one and symmetry reduction in the sense described in [8].

A more abstract (and general) treatment is given elsewhere [12]; in the present note we adopt an approach and notation aimed at applications, and discuss a number of concrete applications. We focus on systems in normal form (sect.2) and in particular on the embedding and splitting of systems with infinitely many resonances (sect.3). Some simple examples are discussed in sect.4, while sect.5 is devoted to (in general non-hamiltonian) nonlinearly perturbed oscillators, and sect. 6 to bifurcation problems.

\section{Equations in normal form; resonances}

We consider an ODE in $R^{n}$ with a fixed point in the origin and expanded around this in a power series; we write this in the form

$$
\frac{\mathrm{d} x}{\mathrm{~d} t}=f(x)=A x+\sum_{k=1}^{\infty} f_{k}(x)
$$

where $f_{k}$ is polynomial with $f_{k}(a x)=a^{k+1}(x)$, and we have singled out the linear part $f_{0}(x)=A x$. We also write $F$ for the nonlinear part of $f$, i.e. $f^{i}(x)=A_{j}^{i} x^{j}+F^{i}(x)$.

\footnotetext{
${ }^{1}$ Another classical instance of simplification by dimension increase is the trick to linearize the matrix Riccati equation. See also $[4,18]$ in this context.

${ }^{2}$ For a different use of the splitting approach in normal forms type problems, see [22].
} 
As well known, the matrix $A$ can be uniquely decomposed into its semisimple and nilpotent parts, which commute with each other and hence with $A$ :

$$
A=A_{s}+A_{n} ;\left[A_{s}, A_{n}\right]=0 .
$$

In the following, we will use the following vector fields $\left(\partial_{i}:=\partial / \partial x^{i}\right)$ :

$$
X_{A}:=(A x)^{i} \partial_{i}, X_{0}=\left(A_{s}\right)^{i} \partial_{i}, X_{F}=F^{i}(x) \partial_{i}, X_{f}=f^{i}(x) \partial_{i} .
$$

\subsection{Resonances}

Let us denote by $\left\{\lambda_{1}, \ldots, \lambda_{n}\right\}$ the eigenvalues of $A$; take a basis $\left\{\mathbf{e}_{1}, \ldots, \mathbf{e}_{n}\right\}$ in $R^{n}$ consisting of generalized eigenvectors of $A$, i.e. eigenvectors of $A_{s}: A_{s} \mathbf{e}_{j}=\lambda_{j} \mathbf{e}_{j}$. We will use $x$ coordinates in this basis, and the multiindex notation

$$
x^{\mu}:=x_{1}^{\mu_{1}} \ldots x_{n}^{\mu_{n}} .
$$

We say that the vector monomial $\mathbf{v}_{\mu, \alpha}:=x^{\mu} \mathbf{e}_{\alpha}$ is resonant with $A$ if

$$
(\mu \cdot \lambda):=\sum_{i=1}^{n} \mu_{i} \lambda_{i}=\lambda_{\alpha} \text { with } \mu_{i} \geq 0,|\mu|:=\sum_{i=1}^{n} \mu_{i} \geq 1 .
$$

The relation $(\mu \cdot \lambda)=\lambda_{\alpha}$ is said to be a resonance relation related to the eigenvalue $\lambda_{\alpha}$, and the integer $|\mu|$ is said to be the order of the resonance. In our context it is useful to include order one resonances in the definition (albeit the trivial order one resonances given by $\lambda_{\alpha}=\lambda_{\alpha}$ are of little interest). Note that here one could as well consider $A_{s}$ rather than $A$.

The space of vectors resonant with (the semisimple part of) $A$ is defined as the linear span of the vectors $\mathbf{v}_{\mu, \alpha}$ defined above.

\subsection{Normal forms}

We say (see e.g. $[1,5,20]$ ) that (1) is in Poincaré-Dulac normal form ${ }^{3}$ if its nonlinear part $F(x)$ is resonant with $A$. This implies that

$$
\left[X_{0}, X_{F}\right]=0 .
$$

It should be mentioned that the presence of a nilpotent part $A_{n}$ in $A$ introduces some subtleties. If (3) holds, then both $X_{A}$ and $X_{F}$ commute with $X_{0}$, and therefore

$$
\left[X_{0}, X_{f}\right]=0
$$

i.e. the system has a symmetry described by a semisimple matrix.

As well known, starting from any dynamical system (or vector field) of the form (1), we can arrive at a dynamical system (or vector field) in Poincaré-Dulac normal form by means of a sequence (in general, infinite) of near-identity transformations obtained by means of the Poincaré algorithm; these combine into a near-identity transformation $H$ defined by a series which is in general only formal.

\footnotetext{
${ }^{3}$ The reader should be warned that some different definition is also in use [7].
} 
Remark 1. We may reformulate the definition of systems in normal form by saying that $f$ is is normal form if and only if $X_{F}$ is in the centralizer of $X_{0}$. $\odot$

Remark 2. If the system is required to have some symmetry, say $\left[X_{f}, X_{g}\right]=0$ with (in an obvious notation) $g^{i}(x)=B_{j}^{i} x^{j}+G^{i}$, then $\left[X_{f}, X_{B}\right]=0$ as well, i.e. $X_{F}$ is in the centralizers of both $X_{0}$ and $X_{B}$. More generally, if $X_{f}$ is an element of some Lie algebra $\mathcal{G}$, then under suitable conditions, see [5], it can be put in joint normal form, i.e. (again with obvious notations) $\left[X_{f}, X_{B_{i}}\right]=0 . \odot$

\subsection{Sporadic resonances and invariance relations}

Let us consider again the resonance equation (2). It is clear that if there are non-negative integers $\sigma_{i}$ (some of them nonzero) such that

$$
\sum_{i=1}^{n} \sigma_{i} \lambda_{i}=0
$$

then we always have infinitely many resonances. The monomial $\phi=x^{\sigma}$ will be called a resonant scalar monomial. It is an invariant of $X_{0}$, and any multiindex $\mu$ with $\mu_{i}=k \sigma_{i}+\delta_{i \alpha}$ provides a resonance relation $(\mu \cdot \lambda)=\lambda_{\alpha}$ related to the eigenvalue $\lambda_{\alpha}$; in other words, any monomial $x^{k \sigma} x^{\alpha}=\phi x^{\alpha}$ is resonant, and so is any vector $\mathbf{v}_{k \sigma+e_{\alpha}, \alpha}$.

Therefore, we say that (5) identifies a invariance relation. The presence of invariance relations is the only way to have infinitely many resonances in a finite dimensional system (see $[20])$.

Any nontrivial resonance (2) such that there is no $\sigma$ with $\sigma_{i} \leq \mu_{i}$ (for all $i=1, \ldots, n$ ) providing an invariance relation, is said to be a sporadic resonance. Sporadic resonances are always in finite number (if any) in a finite dimensional system [20].

Any invariance relation (5) such that there is no $\nu$ with $\nu_{i} \leq \sigma_{i}$ (and of course $\nu \neq \sigma$ ) providing another invariance relation, is said to be an elementary invariance relation. Every invariance relation is a linear combination (with nonnegative integer coefficients) of elementary ones. Elementary invariance relations are always in finite number (if any) in a finite dimensional system [20].

\section{Embedding systems with invariance relations in quasi- linear systems}

In this section we will discuss how the procedure described in [10] generalizes, in connection with a "splitting" of the system described by $Y$ [8], in the presence of invariance relations.

We should preliminarily identify all sporadic resonances $(\mu \cdot \lambda)=\lambda_{\alpha}$ and elementary invariance relations $(\sigma \cdot \lambda)=0$. We associate resonant monomials $x^{\mu}$ and resonant vectors $\mathbf{v}_{\mu, \alpha}$ to the former ones, and invariant monomials $x^{\sigma}$ to the latter ones.

We then introduce two set of new coordinates: these will be the coordinates $w^{1}, \ldots, w^{r}$ in correspondence with sporadic resonances (as in [10]), and other new coordinates $\phi^{1}, \ldots, \phi^{m}$ in correspondence with elementary invariance relations. 
We should also assign evolution equations for the $w$ and $\phi$ coordinates; these will be given in agreement with (1) itself. That is, the equations for the $w$ will be

$$
\frac{\mathrm{d} w^{j}}{\mathrm{~d} t}=\frac{\partial w^{j}}{\partial x^{i}} \frac{\mathrm{d} x^{i}}{\mathrm{~d} t}:=h^{j}(x, w, \phi) ;
$$

and as for the $\phi$ 's we assign

$$
\frac{\mathrm{d} \phi^{a}}{\mathrm{~d} t}=\frac{\partial \phi^{a}}{\partial x^{i}} \frac{\mathrm{d} x^{i}}{\mathrm{~d} t}:=z^{a}(x, w, \phi) .
$$

We will thus consider the enlarged space $W=(x, w, \phi)=\mathbf{R}^{n+r+m}$, and in this the vector field

$$
Y=f^{i}(x, w, \phi) \frac{\partial}{\partial x^{i}}+h^{j}(x, w, \phi) \frac{\partial}{\partial w^{j}}+z^{a}(x, w, \phi) \frac{\partial}{\partial \phi^{a}} .
$$

Note that some ambiguity is present here, in that we can write the coefficients of this vector field in different ways as a function of the $x, w, \phi$. Indeed, the vector field $Y$ is uniquely defined only on the manifold identified by $\psi^{i}:=w^{i}-x^{\mu_{(i)}}=0, \phi_{a}-\zeta_{a}(x)=0$.

Lemma 1. The $(n+m)$-dimensional manifold $M \subset W$ identified by $\psi^{i}:=w^{i}-x^{\mu^{(i)}}=0$ is invariant under the flow of $Y$.

Proof. Obvious by construction.

Lemma 2. The functions $z^{a}$ defined in (7) can be written in terms of the $\phi$ variables alone, i.e. $\partial z^{a} / \partial x^{i}=\partial z^{a} / \partial w^{j}=0$.

Proof. Every analytic invariant of $X_{0}$ can be represented as a convergent series in the $z^{a}$ (see [20]); their evolution is also invariant under the $X_{0}$ action, hence can also be written in terms of invariants, hence of the $z^{a}$ themselves. $\triangle$

Corollary 1. The evolution of the $\phi$ variables is described by a (nonlinear) equation in the $\phi$ variables only.

Proof. This is merely a restatement of lemma 2 above. Note that the equations for $x$ and $w$ depend on $\phi$ and are therefore nonautonomous.

Proposition 3. The analytic functions $f^{i}$ and $h^{j}$ defined above can be written as linear in the $x$ and $w$ variables, the coefficients being functions of the $\phi$ variables.

Proof. Recall each $w^{j}$ is a monomial $w^{j}=x_{1}^{p_{1}} \ldots x_{n}^{p_{n}}$ with $(p, \lambda)=\lambda_{s}$ for some $s=1, \ldots, n$; with reference to this integer $s$, we add a label to $w_{j}$, i.e. write $w_{j}^{(s)}$. By construction and by the results above, each $f^{m}$ can be written in the form $f^{m}=a^{m}(\phi) \cdot x_{m}+\sum_{k} c_{k}^{m}(\phi) w_{k}^{(m)}$, with analytic $a_{m}$ and $c_{k}^{m}$. So the assertion for the $f^{m}$ is obvious.

The time derivative of $w_{j}^{(s)}$ under the flow of (1) will be $\dot{w}_{j}^{(s)}=\left(\partial w_{j}^{(s)} / \partial x^{m}\right) f^{m}$. Therefore it is sufficient to show that $\left(\partial w_{j}^{(s)} / \partial x^{m}\right) w_{k}^{(m)}$ is zero or a multiple of $x^{m}$ or of some $w_{\ell}^{(s)}$ with a suitable resonant scalar monomial as a factor. But the above operation just means to replace one factor $x^{m}$ by $w_{k}^{(m)}$, and the resulting linear combination of the eigenvalues still yields a resonance relation (2) with $\lambda_{s}$ on the right hand side. See [12] for a different approach to the proof. $\triangle$ 
Corollary 2. The evolution of the $x$ and $w$ variables is described by nonautonomous linear equations, obtained by inserting the solution $\phi=\phi(t)$ of the equations for $\phi$ in the general equations $\dot{x}=f(x, w, \phi), \dot{w}=h(x, w, \phi)$.

Proof. Obvious. $\triangle$

Remark 3. We will also say that the vector field $Y$ is quasi-linear, meaning by this that it is linear in the $x$ and $w$ variables. In this way we recover - as a special case - the situation discussed in [9] as well as the terminology used there. $\odot$

Remark 4. The results obtained here extend and unify those given in [8, 10]; see also [20]. As the $\phi$ identify group orbits for the group $G$ generated by the Lie algebra, we interpret $\dot{\phi}=z(\phi)$ as an equation in orbit space, and the equation for $(x, w)$ as an equation on the Lie group G. Methods for the solution of the latter are discussed in [21], see also [4]. $\odot$

Remark 5. If no invariance relations are present, hence no $\phi$ variables are introduced, then the system describing the time evolution of the $x, w$ variables is linear; this is the situation studied in [10]. Note that in this case we have exactly the interpretation of normal forms as projection of a linear system to an invariant manifold, without symmetry reduction. $\odot$

Remark 6. If there are no sporadic resonances of order greater than one then Proposition 3 yields a linear system for the $f^{i}$, with functions of the $\phi$ variables as coefficients. Therefore, upon solving the reduced equation for the $\phi$ variables one obtains a non-autonomous linear system. Moreover, if all eigenvalues are distinct then we have a product system of one-dimensional equations. $\odot$

Remark 7. Finally, we note that if $\phi(t)$ converges to some $\phi_{0}$ (this is always the case if the $\phi$ space is one-dimensional and $|\phi(t)|$ does not escape to infinity), the asymptotic evolution of the system is governed by a linear autonomous equation for $x$ and $w$ (see [19] for the behavior of asymptotically autonomous equations). Similarly, if there is a periodic solution $\bar{\phi}(t)$ with $\phi(t) \rightarrow \bar{\phi}(t)$, the asymptotic evolution of the system is governed by a linear equation with periodic coefficients for $x$ and $w$. $\odot$

\section{Examples}

Example 1. (See [10]). For $A=\operatorname{diag}(1, k), k \in \mathbf{N}$, the only resonant vector is $\mathbf{v}=x^{k} \mathbf{e}_{2}$, corresponding to a sporadic resonance, and there is no invariance relation. Systems in normal form correspond to

$$
X=x \partial_{x}+\left(k y+c x^{k}\right) \partial_{y}
$$

with $c$ a real constant. According to our procedure, we define $w=x^{k}$, and obtain

$$
Y=x \partial_{x}+(k y+c w) \partial_{y}+k w \partial_{w}
$$

the invariant manifold $M$ is given by $\psi:=w-x^{k}=0$. The solution to the system in $W$ for initial data $\left(x_{0}, y_{0}, w_{0}\right)$ is $x(t)=x_{0} e^{t}, y(t)=y_{0} e^{k t}+\left(c_{1} k w_{0}\right) t e^{k t}, w(t)=w_{0} e^{k t}$; for initial data on $M$, i.e. $w_{0}=x_{0}^{k}$, the solution remains on $M$ and its projection to $\mathbf{R}^{2}=(x, y)$ is $x(t)=x_{0} e^{t}, y(t)=\left[y_{0}+\left(c_{1} k x_{0}^{k}\right) t\right] e^{k t}$. 
Example 2. Consider the matrix

$$
A=\left(\begin{array}{ccc}
0 & -1 & 0 \\
1 & 0 & 0 \\
0 & 0 & 1
\end{array}\right)
$$

with eigenvalues $(-i, i, 1)$. There is one elementary invariance relation, $\lambda_{1}+\lambda_{2}=0$, and no sporadic resonance. The linear centralizer is spanned by $A$ itself and by matrices $D_{1}=$ $\operatorname{diag}(1,1,0)$ and $D_{2}=\operatorname{diag}(0,0,1)$. The ring of invariants is generated by $r^{2}:=x^{2}+y^{2}$. Systems in normal forms are written as

$$
\begin{aligned}
& \dot{x}=\alpha\left(r^{2}\right) x-\beta\left(r^{2}\right) y \\
& \dot{y}=\beta\left(r^{2}\right) x+\alpha\left(r^{2}\right) y \\
& \dot{z}=\gamma\left(r^{2}\right) z
\end{aligned}
$$

where $\alpha, \beta, \gamma$ are arbitrary power series.

Following our procedure, we introduce one further variable $\phi=r^{2}$; for this we have $d \phi / d t=2(x \dot{x}+y \dot{y})=2 r^{2} \alpha\left(r^{2}\right)$. Hence the system in $W=\mathbf{R}^{4}$ is written as

$$
\begin{aligned}
& \dot{x}=\alpha(\phi) x-\beta(\phi) y \\
& \dot{y}=\beta(\phi) x+\alpha(\phi) y \\
& \dot{z}=\gamma(\phi) z \\
& \dot{\phi}=2 \phi \alpha(\phi) .
\end{aligned}
$$

Example 3. Consider the matrix

$$
A=\left(\begin{array}{cccc}
0 & -1 & 0 & 0 \\
1 & 0 & 0 & 0 \\
0 & 0 & 1 & 0 \\
0 & 0 & 0 & k
\end{array}\right)
$$

( $k \in \mathbf{N}, k>1)$ with eigenvalues $(-i,+i, 1, k)$. There is one sporadic resonance, $k \lambda_{3}=\lambda_{4}$, and one elementary invariance relation, $\lambda_{1}+\lambda_{2}=0$. The linear centralizer is spanned the matrices $D_{1}=\operatorname{diag}(1,1,0,0), D_{2}=\operatorname{diag}(0,0,1,0), D_{3}=\operatorname{diag}(0,0,0,1)$ together with

$$
M_{1}=\left(\begin{array}{cccc}
0 & -1 & 0 & 0 \\
1 & 0 & 0 & 0 \\
0 & 0 & 0 & 0 \\
0 & 0 & 0 & 0
\end{array}\right) \text { and } M_{2}=\left(\begin{array}{cccc}
0 & 0 & 0 & 0 \\
0 & 0 & 0 & 0 \\
0 & 0 & 0 & 0 \\
0 & 0 & k & 0
\end{array}\right)
$$

Systems in normal forms are written as

$$
\begin{aligned}
& \dot{x}_{1}=\alpha\left(r^{2}\right) x_{1}-\beta\left(r^{2}\right) x_{2} \\
& \dot{x}_{2}=\beta\left(r^{2}\right) x_{1}+\alpha\left(r^{2}\right) x_{2} \\
& \dot{x}_{3}=\gamma\left(r^{2}\right) x_{3} \\
& \dot{x}_{4}=\eta\left(r^{2}\right) x_{4}+\theta\left(r^{2}\right) x_{3}^{k}
\end{aligned}
$$

where $r^{2}:=x^{2}+y^{2}$ and $\alpha, \beta, \gamma, \eta, \theta$ are arbitrary power series. 
Following our procedure we introduce $\phi=r^{2}$, for which $d \phi / d t=2(x \dot{x}+y \dot{y})=2 r^{2} \alpha\left(r^{2}\right)$, and $w=x_{3}^{k}$ for which $d w / d t=k x_{3}^{k-1} \dot{x}_{3}=k \gamma\left(r^{2}\right) x_{3}^{k}$. Hence the system in $W=\mathbf{R}^{5}$ is written as

$$
\begin{aligned}
& \dot{x}_{1}=\alpha(\phi) x_{1}-\beta(\phi) x_{2} \\
& \dot{x}_{2}=\beta(\phi) x_{1}+\alpha(\phi) x_{2} \\
& \dot{x}_{3}=\gamma(\phi) x_{3} \\
& \dot{w}=k \gamma(\phi) w \\
& \dot{\phi}=2 \phi \alpha(\phi) .
\end{aligned}
$$

\section{$5 \quad$ Perturbed oscillators}

Example 4. Perturbation of oscillators in 1:1 resonance. Consider the matrix

$$
A=\left(\begin{array}{cccc}
0 & -1 & 0 & 0 \\
1 & 0 & 0 & 0 \\
0 & 0 & 0 & -1 \\
0 & 0 & 1 & 0
\end{array}\right)
$$

which we also write in block form as

$$
A=\left(\begin{array}{ll}
J & 0 \\
0 & J
\end{array}\right), \text { where } J=\left(\begin{array}{cc}
0 & -1 \\
1 & 0
\end{array}\right),
$$

with eigenvalues $(-i,+i,-i, i)$. There are no sporadic resonances of order greater than one, and four elementary invariance relations:

$$
\lambda_{1}+\lambda_{2}=0, \lambda_{3}+\lambda_{4}=0, \lambda_{1}+\lambda_{4}=0, \lambda_{2}+\lambda_{3}=0 ;
$$

all other resonances can be described in terms of these. We stress that the equations describing these invariance relations are linearly dependent; however they should be considered, according to our definition, as different elementary ones. Corresponding to this, the associated invariant quantities will not be functionally independent (obviously, we cannot have more than three independent invariants for a flow in $\mathbf{R}^{4}$ ).

The linear centralizer of $A$ is an eight-dimensional algebra, spanned by the following matrices (in two by two block notation):

$$
\begin{gathered}
B_{1}=\left(\begin{array}{ll}
I & 0 \\
0 & 0
\end{array}\right), B_{2}=\left(\begin{array}{ll}
0 & 0 \\
0 & I
\end{array}\right), B_{3}=\left(\begin{array}{cc}
0 & I \\
I & 0
\end{array}\right), B_{4}=\left(\begin{array}{cc}
0 & J \\
-J & 0
\end{array}\right), \\
S_{1}=\left(\begin{array}{ll}
J & 0 \\
0 & 0
\end{array}\right), S_{2}=\left(\begin{array}{ll}
0 & 0 \\
0 & J
\end{array}\right), S_{3}=\left(\begin{array}{cc}
0 & I \\
-I & 0
\end{array}\right), S_{4}=\left(\begin{array}{cc}
0 & J \\
J & 0
\end{array}\right) .
\end{gathered}
$$

Note here we have chosen a basis with $B_{i}=B_{i}^{+}, S_{i}=-S_{i}^{+}$.

The linear system $\dot{\xi}=A \xi$ describes two oscillators in 1:1 resonance; the normal form will correspond to a perturbation of these, generically breaking the exchange symmetry among the two oscillators. Systems in normal form are compactly written as

$$
\left(\begin{array}{c}
\dot{x} \\
\dot{y} \\
\dot{z} \\
\dot{w}
\end{array}\right)=\left(\begin{array}{cccc}
\alpha & -\beta & \gamma & -\eta \\
\beta & \alpha & \eta & \gamma \\
\mu & -\nu & \sigma & -\tau \\
\nu & \mu & \tau & \sigma
\end{array}\right)\left(\begin{array}{c}
x \\
y \\
z \\
w
\end{array}\right)
$$


where $\alpha, \beta, \ldots, \tau$ are arbitrary power series in the elementary invariants

$$
\phi_{1}=x^{2}+y^{2}, \phi_{2}=z^{2}+w^{2}, \phi_{3}=x z+y w, \phi_{4}=x w-y z ;
$$

note that $\phi_{a}=\left(\xi, B_{a} \xi\right)$, with (.,.) the scalar product. We abbreviate the above evolution equation as

$$
\dot{\xi}=K(\phi) \xi .
$$

The evolution equations for the $\phi$, as required by our procedure, are simply (only the selfadjoint matrices defined in (9) appear)

$$
\dot{\phi}_{a}=\left(\xi,\left(B_{a} K+K^{+} B_{a}\right) \xi\right) .
$$

Example 5. Perturbation of oscillators in $1: k$ resonance. Consider the matrix

$$
A=\left(\begin{array}{cccc}
0 & -1 & 0 & 0 \\
1 & 0 & 0 & 0 \\
0 & 0 & 0 & -k \\
0 & 0 & k & 0
\end{array}\right)
$$

(with $k \in \mathbf{N}, k>1$ ) with eigenvalues $(-i,+i,-i k, i k)$. This is put in diagonal form passing to variables

$$
\xi_{1}=\left(x_{1}-i x_{2}\right) / 2, \xi_{2}=\left(x_{1}+i x_{2}\right) / 2, \xi_{3}=\left(x_{3}-i x_{4}\right) / 2, \xi_{4}=\left(x_{3}+i x_{4}\right) / 2,
$$

which we use in intermediate computations below. We also write $\xi=\Lambda x, x=\Lambda^{-1} \xi$, with

$$
\Lambda=\frac{1}{2}\left(\begin{array}{cccc}
1 & -i & 0 & 0 \\
1 & 1 & 0 & 0 \\
0 & 0 & 1 & -i \\
0 & 0 & 1 & i
\end{array}\right) ; \Lambda^{-1}=\left(\begin{array}{cccc}
1 & 1 & 0 & 0 \\
i & -i & 0 & 0 \\
0 & 0 & 1 & 1 \\
0 & 0 & i & -i
\end{array}\right)
$$

There are four sporadic resonances:

$$
k \lambda_{1}=\lambda_{3}, k \lambda_{2}=\lambda_{4}, \lambda_{3}+(k-1) \lambda_{2}=\lambda_{1}, \lambda_{4}+(k-1) \lambda_{1}=\lambda_{2} .
$$

Moreover, there are four elementary invariance relations:

$$
\lambda_{1}+\lambda_{2}=0, \lambda_{3}+\lambda_{4}=0, k \lambda_{1}+\lambda_{4}=0, k \lambda_{2}+\lambda_{3}=0 .
$$

All other resonances can be described in terms of these.

Systems in normal forms are written as

$$
\begin{aligned}
& \dot{\xi}_{1}=\alpha_{1} \xi_{1}+\vartheta_{1} \xi_{2}^{k-1} \xi_{3} \\
& \dot{\xi}_{2}=\alpha_{2} \xi_{2}+\vartheta_{2} \xi_{1}^{k-1} \xi_{4} \\
& \dot{\xi}_{3}=\alpha_{3} \xi_{3}+\vartheta_{3} \xi_{1}^{k} \\
& \dot{\xi}_{4}=\alpha_{4} \xi_{4}+\vartheta_{4} \xi_{2}^{k}
\end{aligned}
$$

where $\alpha_{i}, \vartheta_{i}$ are arbitrary power series in the invariants of the linear flow. 
Following our procedure, we introduce variables

$$
w_{1}=\xi_{1}^{k}, w_{2}=\xi_{2}^{k}, w_{3}=\xi_{2}^{k-1} \xi_{3}, w_{4}=\xi_{1}^{k-1} \xi_{4},
$$

related to sporadic resonances. We also introduce variables related to elementary invariance relations, given by

$$
\phi_{1}=\xi_{1} \xi_{2}, \phi_{2}=\xi_{3} \xi_{4}, \phi_{3}=\xi_{1}^{k} \xi_{4}, \phi_{4}=\xi_{2}^{k} \xi_{3} .
$$

We must then introduce evolution equations for the $w$ and $\phi$ variables according to our procedure, i.e. according to (6) and (7) above. As for the $w$, we get

$$
\begin{aligned}
& \dot{w}_{1}=k \alpha_{1} w_{1}+\vartheta_{1} \phi_{1}^{k-1} \xi_{3} \\
& \dot{w}_{2}=k \alpha_{2} w_{2}+\vartheta_{2} \phi_{1}^{k-1} \xi_{4} \\
& \dot{w}_{3}=\left[\alpha_{3}+(k-1) \alpha_{2}\right] w_{3}+\left[(k-1) \vartheta_{2} \phi_{1}^{k-2} \phi_{2}+\vartheta_{3} \phi_{1}^{k-1}\right] \xi_{1} \\
& \dot{w}_{4}=\left[\alpha_{4}+(k-1) \alpha_{2}\right] w_{4}+\left[(k-1) \vartheta_{1} \phi_{1}^{k-2} \phi_{2}+\vartheta_{4} \phi_{1}^{k-1}\right] \xi_{2} .
\end{aligned}
$$

Let us now consider the equations for the $\phi$; we easily get

$$
\begin{aligned}
\dot{\phi}_{1} & =\left(\alpha_{1}+\alpha_{2}\right) \phi_{1}+\vartheta_{2} \phi_{3}+\vartheta_{1} \phi_{4} \\
\dot{\phi}_{2} & =\left(\alpha_{3}+\alpha_{4}\right) \phi_{2}+\vartheta_{3} \phi_{3}+\vartheta_{4} \phi_{4} \\
\dot{\phi}_{3} & =\left(k \alpha_{1}+\alpha_{4}\right) \phi_{3}+k \vartheta_{1} \phi_{1}^{k-1} \phi_{2}+\vartheta_{4} \phi_{1}^{k} \\
\dot{\phi}_{4} & =\left(k \alpha_{2}+\alpha_{3}\right) \phi_{4}+k \vartheta_{2} \phi_{1}^{k-1} \phi_{2}+\vartheta_{3} \phi_{1}^{k} .
\end{aligned}
$$

Summarizing, all systems of the form (10) - i.e. in normal form with respect to the linear part $\dot{\xi}=A \xi$ - are written as the autonomous system (11) for the $\phi$ variables, plus a linear nonautonomous system, which introducing the notation $\eta=(\xi ; w)$ can be written as

$$
\dot{\eta}=M \eta
$$

where $M=M(\phi)$ is a matrix which we write as $M=D+L$, where

$$
D=\operatorname{diag}\left(\alpha_{1}, \alpha_{2}, \alpha_{3}, \alpha_{4} ; k \alpha_{1}, k \alpha_{2}, \alpha_{3}+(k-1) \alpha_{2}, \alpha_{4}+(k-1) \alpha_{1}\right)
$$

and $L$ is an off-diagonal sparse matrix with nonzero terms

$$
\begin{gathered}
L_{17}=\vartheta_{1}, L_{28}=\vartheta_{2}, L_{35}=\vartheta_{3}, L_{46}=\vartheta_{4} ; L_{53}=\vartheta_{1} \phi_{1}^{k-1}, L_{64}=\vartheta_{2} \phi_{1}^{k-1} \\
L_{71}=\left[(k-1) \vartheta_{2} \phi_{1}^{k-2} \phi_{2}+\vartheta_{3} \phi_{1}^{k-1}\right], L_{82}=\left[(k-1) \vartheta_{1} \phi_{1}^{k-2} \phi_{2}+\vartheta_{4} \phi_{1}^{k-1}\right] .
\end{gathered}
$$

Example 6. Perturbation of two oscillators with no resonance. Consider the matrix

$$
A=\left(\begin{array}{cccc}
0 & -1 & 0 & 0 \\
1 & 0 & 0 & 0 \\
0 & 0 & 0 & -\pi \\
0 & 0 & \pi & 0
\end{array}\right)
$$

with eigenvalues $(-i,+i,-\pi i, \pi i)$.

Now there are no sporadic resonances of order greater than one, and two elementary invariance relations:

$$
\lambda_{1}+\lambda_{2}=0, \lambda_{3}+\lambda_{4}=0 .
$$


Note that now we have only two invariants, $r_{1}^{2}=x_{1}^{2}+x_{2}^{2}$ and $r_{2}^{2}=x_{3}^{2}+x_{4}^{2}$ : this is easily understood as we have irrational flow on the two-torus $\mathbf{T}^{2} \subset \mathbf{R}^{4}$. The centralizer of $A$ corresponds to matrices

$$
M=\left(\begin{array}{cccc}
a & -b & 0 & 0 \\
b & a & 0 & 0 \\
0 & 0 & c & -d \\
0 & 0 & d & c
\end{array}\right)
$$

Thus systems in normal form will be written as

$$
\left(\begin{array}{c}
\dot{x}_{1} \\
\dot{x}_{2} \\
\dot{x}_{3} \\
\dot{x}_{4}
\end{array}\right)=\left(\begin{array}{cccc}
\alpha & -\beta & 0 & 0 \\
\beta & \alpha & 0 & 0 \\
0 & 0 & \gamma & -\eta \\
0 & 0 & \eta & \gamma
\end{array}\right)\left(\begin{array}{l}
x_{1} \\
x_{2} \\
x_{3} \\
x_{4}
\end{array}\right)
$$

with $\alpha, \beta, \gamma, \eta$ being power series in $r_{1}^{2}, r_{2}^{2}$.

Following our procedure we introduce variables $\phi_{1}=x_{1}^{2}+x_{2}^{2}$ and $\phi_{2}=x_{3}^{2}+x_{4}^{2}$, whose evolution is given by

$$
\dot{\phi}_{1}=2 \alpha \phi_{1}, \dot{\phi}_{2}=2 \gamma \phi_{2} \text {. }
$$

Example 7. Perturbation of oscillators in 1:1:1 resonance. Consider the sixdimensional matrix written in block form as

$$
A=\left(\begin{array}{ccc}
\omega J & 0 & 0 \\
0 & \omega J & 0 \\
0 & 0 & \omega J
\end{array}\right) \text { where } J=\left(\begin{array}{cc}
0 & -1 \\
1 & 0
\end{array}\right) \text {. }
$$

Passing to coordinates $\left(\xi_{j}, \eta_{j}\right)=\left(p_{j}+i q_{j}, p_{j}-i q_{j}\right)$, this reads

$$
\widehat{A}=\operatorname{diag}(i \omega,-i \omega, i \omega,-i \omega, i \omega,-i \omega) .
$$

The eigenvalues are $\lambda_{k}=(-1)^{k} i \omega$, hence there is no sporadic resonance of order greater than one $e^{4}$ and nine invariance relations:

$$
\begin{array}{lll}
\lambda_{1}+\lambda_{2}=0 & \lambda_{3}+\lambda_{4}=0 & \lambda_{5}+\lambda_{6}=0 \\
\lambda_{1}+\lambda_{4}=0 & \lambda_{1}+\lambda_{6}=0 & \lambda_{3}+\lambda_{6}=0 \\
\lambda_{2}+\lambda_{3}=0 & \lambda_{2}+\lambda_{5}=0 & \lambda_{4}+\lambda_{5}=0
\end{array}
$$

The invariants corresponding to these are of course

$$
\begin{array}{lll}
\widehat{\phi}_{1}=\xi_{1} \eta_{1}, & \widehat{\phi}_{2}=\xi_{2} \eta_{2}, & \widehat{\phi}_{3}=\xi_{3} \eta_{3}, \\
\widehat{\phi}_{4}=\xi_{1} \eta_{2}, & \widehat{\phi}_{5}=\xi_{1} \eta_{3}, & \widehat{\phi}_{6}=\xi_{2} \eta_{3}, \\
\widehat{\phi}_{7}=\xi_{2} \eta_{1}, & \widehat{\phi}_{8}=\xi_{3} \eta_{2}, & \widehat{\phi}_{9}=\xi_{3} \eta_{2} .
\end{array}
$$

Going back to the original coordinates, expressions for these are recovered from (no sum on repeated indices)

$$
\xi_{j} \eta_{j}=\left(p_{j}^{2}+q_{j}^{2}\right) ; \xi_{j} \eta_{k}=\left(p_{j} p_{k}+q_{j} q_{k}\right)+i\left(q_{j} p_{k}-p_{j} q_{k}\right)
$$

\footnotetext{
${ }^{4}$ Note these are present in the case of $1: k: \ell$ resonance (with integers $k, \ell>1$ ).
} 
and is thus more convenient to pass to a different basis for invariant functions, i.e.

$$
\begin{array}{ll}
\phi_{1}:=\widehat{\phi}_{1}=\left(p_{1}^{2}+q_{1}^{2}\right), \phi_{2}:=\widehat{\phi}_{2}=\left(p_{2}^{2}+q_{2}^{2}\right), \quad \phi_{3}:=\widehat{\phi}_{3}=\left(p_{3}^{2}+q_{3}^{2}\right) ; \\
\phi_{4}:=\left(\widehat{\phi}_{4}+\widehat{\phi}_{7}\right) / 2=\left(p_{1} p_{2}+q_{1} q_{2}\right), \quad \phi_{5}:=\left(\widehat{\phi}_{4}-\widehat{\phi}_{7}\right) /(2 i)=\left(q_{1} p_{2}-p_{1} q_{2}\right) ; \\
\phi_{6}:=\left(\widehat{\phi}_{5}+\widehat{\phi}_{9}\right) / 2=\left(p_{1} p_{3}+q_{1} q_{3}\right) \quad, \quad \phi_{7}:=\left(\widehat{\phi}_{5}-\widehat{\phi}_{9}\right) /(2 i)=\left(q_{1} p_{3}-p_{1} q_{3}\right) ; \\
\phi_{8}:=\left(\widehat{\phi}_{6}+\widehat{\phi}_{8}\right) / 2=\left(p_{2} p_{3}+q_{2} q_{3}\right), \quad \phi_{9}:=\left(\widehat{\phi}_{6}-\widehat{\phi}_{8}\right) /(2 i)=\left(q_{2} p_{3}-p_{2} q_{3}\right) .
\end{array}
$$

The centralizer $C(A)$ of $A$ in $\operatorname{Mat}(6, R)$ is an algebra spanned by eighteen matrices; these are written in $2 \times 2$ block form as

$$
\left(\begin{array}{lll}
C_{11} & C_{12} & C_{13} \\
C_{21} & C_{22} & C_{23} \\
C_{31} & C_{32} & C_{33}
\end{array}\right)
$$

where the $C_{i j}$ are two-dimensional matrices written as $\left(\alpha_{i j}\right.$ and $\beta_{i j}$ real constants)

$$
C_{i j}=\alpha_{i j} I+\beta_{i j} J .
$$

It is easy to extract from these a basis made of nine selfadjoint matrices $B_{k}=B_{k}^{+}$and nine antiselfadjoint ones $S_{k}=-S_{k}^{+}$. With $\mathbf{x}=\left(p_{1}, q_{1}, p_{2}, q_{2}, p_{3}, q_{3}\right)$, and (., .) the standard scalar product in $\mathbf{R}^{6}$ these can be chosen so that $\phi_{a}=\left(\mathbf{x}, B_{a} \mathbf{x}\right)$.

In this compact notation the $1: 1: 1$ resonant three-dimensional oscillator is described by $\dot{\mathbf{x}}=A \mathbf{x}$. We write the normal form of evolution equations for perturbation of this as

$$
\begin{aligned}
\dot{\mathbf{x}} & =K(\phi) \mathbf{x} \\
\dot{\phi}_{a} & =\left(\mathbf{x},\left(B_{a} K+K^{+} B_{a}\right) \mathbf{x}\right)
\end{aligned}
$$

with $K$ an arbitrary matrix in $C(A)$.

\section{Bifurcations}

Example 8. Hopf bifurcation. Consider the matrix

$$
A=\left(\begin{array}{cc}
0 & -\omega_{0} \\
\omega_{0} & 0
\end{array}\right)
$$

with eigenvalues $\lambda_{1}=-i \omega_{0}, \lambda_{2}=i \omega_{0}$. There is no sporadic resonance, and one elementary invariance relation, $\lambda_{1}+\lambda_{2}=0$, with associated invariant $\phi=x^{2}+y^{2}$. The most general system in normal form is therefore $\dot{x}=\alpha(\phi) x-\beta(\phi) y, \dot{y}=\beta(\phi) x+\alpha(\phi) y$. According to our procedure, the evolution equation for the new coordinate $\phi$ will be $\dot{\phi}=2 \phi \alpha(\phi)$. As the linear part of the system is given by $A$, we must require $\alpha(0)=0, \beta(0)=\omega_{0}$.

In applications, one is interested in the case where the system does also depend on an external ("control") parameter $\mu$, which usually does not evolve in time ${ }^{5}$, the linear part being given by $A$ at the critical value. In this case the normal form and the $\phi$ evolution equation read

$$
\begin{aligned}
& \dot{x}=\alpha(\phi, \mu) x-\beta(\phi, \mu) y \\
& \dot{y}=\beta(\phi, \mu) x+\alpha(\phi, \mu) y \\
& \dot{\phi}=2 \phi \alpha(\phi, \mu) .
\end{aligned}
$$

${ }^{5} \mathrm{~A}$ different framework is provided by dynamic bifurcations $[2,17]$. 
In the standard model of Hopf bifurcation, $\alpha(\phi, \mu)=\mu-c \phi$, and we write $\beta(\phi, \mu)=$ $\omega_{0}+b(\phi, \mu)$ with $b(0,0)=0$. This corresponds to the normal form

$$
\left\{\begin{array}{l}
\dot{x}=\mu x-\omega_{0} y-b\left(x^{2}+y^{2}, \mu\right) y-c\left(x^{2}+y^{2}\right) x \\
\dot{y}=\omega_{0} x+\mu y+b\left(x^{2}+y^{2}, \mu\right) x-c\left(x^{2}+y^{2}\right) x
\end{array}\right.
$$

which in our approach reads

$$
\left\{\begin{array}{l}
\dot{x}=\mu x-\omega_{0} y-b(\phi, \mu) y-c(\phi) x \\
\dot{y}=\omega_{0} x+\mu y+b(\phi, \mu) x-c(\phi) x \\
\dot{\phi}=2 \phi \alpha(\phi, \mu) .
\end{array}\right.
$$

Note that the space of invariants is one-dimensional (with the additional constraint $\phi \geq 0$ ); thus, either $\phi(t)$ is unbounded for $t>0$, or it approaches one of the zeroes of the function $\alpha(\phi, \mu)$, say $\phi_{0}$. In this case, the system reduces asymptotically to a linear one:

$$
\left(\begin{array}{l}
\dot{x} \\
\dot{y}
\end{array}\right)=\left(\begin{array}{cc}
0 & -\omega_{0}-b\left(\phi_{0}, \mu\right) \\
\omega_{0}+b\left(\phi_{0}, \mu\right) & 0
\end{array}\right)\left(\begin{array}{l}
x \\
y
\end{array}\right) .
$$

The standard analysis of Hopf bifurcation is readily recovered in this way.

Note that we can also look at Hopf bifurcation in a slightly different way, i.e. include the $\mu$ variable from the beginning. In this case the matrix $A$ is given by

$$
A=\left(\begin{array}{ccc}
0 & -1 & 0 \\
1 & 0 & 0 \\
0 & 0 & 0
\end{array}\right)
$$

with eigenvalues $(-i, i, 0)$ and invariance relations $\lambda_{1}+\lambda_{2}=0$ and $\lambda_{3}=0$; the associated invariants are $\phi$ and $\mu$. The linear centralizer of $A$ is spanned by matrices

$$
M=\left(\begin{array}{ccc}
a & -b & 0 \\
b & a & 0 \\
0 & 0 & c
\end{array}\right)
$$

and correspondingly the normal form will be

$$
\begin{aligned}
& \dot{x}=\alpha(\phi, \mu) x-\beta(\phi, \mu) y \\
& \dot{y}=\beta(\phi, \mu) x+\alpha(\phi, \mu) y \\
& \dot{\mu}=\gamma(\phi, \mu)
\end{aligned}
$$

The equation for $\phi$ is just the one given above, and we are led to the same system; interpreting $\mu$ as an external control parameter forces $\gamma(\phi, \mu) \equiv 0$ (if not, there is a feedback of the system on the control parameter).

Example 9. Hamiltonian Hopf bifurcation. Consider the matrix

$$
A=\left(\begin{array}{cccc}
\mu & -\omega & 0 & 0 \\
\omega & \mu & 0 & 0 \\
0 & 0 & -\mu & -\omega \\
0 & 0 & \omega & -\mu
\end{array}\right)=\left(\begin{array}{cc}
\mu I+\omega J & 0 \\
0 & -\mu I+\omega J
\end{array}\right)
$$


with eigenvalues $\lambda_{1}=-\mu-i \omega, \lambda_{2}=-\mu+i \omega, \lambda_{3}=\mu-i \omega, \lambda_{4}=\mu+i \omega$. We assume $\omega \neq 0$ (so it could be rescaled to $\omega=1$ ) and $\mu \neq 0$; the case $\mu=0$ corresponds to example 4 . In applications, one considers the case where $\mu$ is an external control parameter and studies the changes as this is varied; $\mu=0$ is a critical value.

The matrix $A$ is diagonalized, for all $\mu$, passing to variables $\xi^{i}$ as in example 5 above. The evolution $\dot{x}=A x$ preserves the symplectic structure $\kappa=\mathrm{d} x^{1} \wedge \mathrm{d} x^{3}+\mathrm{d} x^{2} \wedge \mathrm{d} x^{4}$.

It is easy to check, for generic $\mu$, that there is no sporadic resonance and that there are two elementary invariance relations, given by $\lambda_{1}+\lambda_{4}=0$ and $\lambda_{2}+\lambda_{3}=0$. The corresponding invariants will be $\varphi_{1}=\xi^{1} \xi^{4}, \varphi_{2}=\xi^{2} \xi^{3}$; they are complex conjugate, and correspond to real invariants $\phi_{1}=x_{1} x_{3}+x_{2} x_{4}$ and $\phi_{2}=x_{1} x_{4}-x_{2} x_{3}$. These are also written for later reference as $\phi_{a}=(1 / 2)\left(\xi, B_{a} \xi\right)$, with $B_{a}$ obvious four-dimensional symmetric matrices.

The linear centralizer of $A$ (for $\mu \neq 0$ ) is given by matrices written in block form, with $\alpha_{k}$ and $\beta_{k}$ real constants, as

$$
M=\left(\begin{array}{cc}
\alpha_{1} I+\beta_{1} J & 0 \\
0 & \alpha_{2} I+\beta_{2} J
\end{array}\right) .
$$

Correspondingly, systems in normal form will be given by

$$
\dot{x}=M x
$$

where now $\alpha_{k}, \beta_{k}$ will be functions of the invariants $\phi_{1}, \phi_{2}$. Note that such systems in general do not preserve the symplectic form $\kappa$, unless $\alpha_{2}=-\alpha_{1}$ and $\beta_{2}=\beta_{1}$. The evolution of the $\phi$ is given by $\dot{\phi}_{a}=(1 / 2)\left(x,\left(M^{+} B_{a}+B_{a} M\right) x\right)$.

It is convenient to write the system in terms of the two-dimensional vectors $\eta_{1}=$ $\left(x^{1}, x^{2}\right), \eta_{2}=\left(x^{3}, x^{4}\right)$ and $\phi=\left(\phi_{1}, \phi_{2}\right)$. Moreover, we single out the linear part in the functions $\alpha_{k}$ and $\beta_{k}$, writing $\alpha_{k}(\phi)=(-1)^{k+1} \mu+a(\phi), \beta_{k}(\phi)=\omega+b_{k}(\phi)$ with $a_{k}(0)=b_{k}(0)=0$; the smooth functions are $a_{k}, b_{k}$ are arbitrary apart from this constraint, and can also depend on the parameters $\mu$ and $\omega$.

The system is hence described in our approach by the following equations:

$$
\begin{aligned}
& \dot{\eta}_{1}=(\mu I+\omega J) \eta_{1}+\left[a_{1}(\phi) \cdot I-b_{1}(\phi) \cdot J\right] \eta_{1}, \\
& \dot{\eta}_{2}=(-\mu I+\omega J) \eta_{2}+\left[a_{2}(\phi) \cdot I-b_{2}(\phi) \cdot J\right] \eta_{2}, \\
& \dot{\phi}=\left[\left(a_{1}(\phi)+a_{2}(\phi)\right) \cdot I+\left(b_{2}(\phi)-b_{1}(\phi)\right) \cdot J\right] \phi .
\end{aligned}
$$

If $a_{k}, b_{k}$ are such that the system is hamiltonian, the $\phi$ are always strictly invariant, and we are reduced to a linear system on each level set of $\phi$; if the $a_{k}, b_{k}$ are such that the system is not hamiltonian but the (two-dimensional) equation for the $\phi$ satisfies the condition for the existence of a limit cycle, as is often the case in bifurcation problems, then remark 7 applies.

Let us look more closely to the perturbation of the case $\mu=0$; as recalled above, the analysis of this case can be recovered from example 4. More precisely, we can rescale time so that $\omega=1$, and allow the arbitrary functions appearing in the analysis of example 4 to also depend on the parameter $\mu$.

Alternatively, we can proceed as at the end of the previous example, and include the $\mu$ variable from the beginning. With a $2 \oplus 2 \oplus 1$ block notation, we have now

$$
A=\left(\begin{array}{ccc}
\mu I+\omega J & 0 & 0 \\
0 & -\mu I+\omega J & 0 \\
0 & 0 & 0
\end{array}\right)
$$


with linear centralizer

$$
M=\left(\begin{array}{ccc}
\alpha_{1} I+\beta_{1} J & 0 & 0 \\
0 & \alpha_{2} I+\beta_{2} J & 0 \\
0 & 0 & c
\end{array}\right) .
$$

In the normal form, going back to the original time parametrization, we have

$$
\begin{aligned}
& \dot{\eta}_{1}=(\mu I+\omega J) \eta_{1}+\left[\alpha_{1}(\phi, \mu) \cdot I-\beta_{1}(\phi, \mu) \cdot J\right] \eta_{1}, \\
& \dot{\eta}_{2}=(-\mu I+\omega J) \eta_{2}+\left[\alpha_{2}(\phi, \mu) \cdot I-\beta_{2}(\phi, \mu) \cdot J\right] \eta_{2}, \\
& \dot{\phi}=\left[\left(\alpha_{1}(\phi, \mu)+\alpha_{2}(\phi, \mu)\right) \cdot I+\left(\beta_{2}(\phi, \mu)-\beta_{1}(\phi, \mu)\right) \cdot J\right] \phi, \\
& \dot{\mu}=\gamma(\phi, \mu) .
\end{aligned}
$$

Again, interpreting $\mu$ as an external control parameter requires $\gamma(\phi, \mu) \equiv 0$.

\section{Acknowledgements}

This work was started by discussions during a visit by SW in the Department of Mathematics of Università di Milano, and while GG was a guest at the DFG-Graduiertenkolleg "Hierarchie und Symmetrie in Mathematischen Modellen" at RWTH Aachen. We would like to thank these Institutions for support to our work. GG also acknowledges partial support by Fondazione CARIPLO and by GNFM-INdAM.

\section{References}

[1] V.I. Arnold, Geometrical methods in the theory of ordinary differential equations, Springer (Berlin) 1983, $2^{n} d$ ed. 1989

[2] E. Benoit, Dynamic bifurcations (Lect. Notes Math. 1493), Springer, Berlin 1991

[3] F. Calogero, "Solution of the one-dimensional N-body problems with quadratic and/or inversely quadratic pair potentials", J. Math. Phys. 12 (1971), 419-436; "Exactly solvable one-dimensional many-body problems", Lett. N. Cim. 13 (1975), 411-416

[4] J.F. Cariñena, J. Grabowski and G. Marmo, "Lie-Scheffers systems: a geometric approach", Bibliopolis, Napoli 2000; J.F. Cariẽna and A. Ramos, "A new geometric approach to Lie systems and physical applications", Acta Appl. Math. 70 (2002), 43-69

[5] G. Cicogna and G. Gaeta, Symmetry and perturbation theory in nonlinear dynamics, (Lecture Notes in Physics, vol. M57); Springer (Berlin) 1999

[6] H. Dulac, "Solution d'un systéme d'équations differéntiélles dans le voisinage des valeurs singuliérs", Bull. Soc. Math. France 40 (1912), 324-383

[7] C. Elphick et al., "A simple global characterization for normal forms of singular vector fields", Physica D 29 (1987), 95-127; Addendum, Physica D 32 (1988), 488

[8] G. Gaeta, "A splitting lemma for equivariant dynamics", Lett. Math. Phys. 33 (1995), 313-320 
[9] G. Gaeta, "Algorithmic reduction of Poincaré-Dulac normal forms and Lie algebraic structure", Lett. Math. Phys. 57 (2001), 41-60

[10] G. Gaeta, "Resonant Poincaré-Dulac normal forms as constrained linear systems", Mod. Phys. Lett. A 17 (2002), 583-597

[11] G. Gaeta and S. Walcher, "Linear Lie algebras with finite dimensional centralizer", J. Math. Anal. Appl. 269 (2002), 578-587

[12] G. Gaeta and S. Walcher, "Embedding and splitting ordinary differential equations in normal form", preprint 2004

[13] D. Kazhdan, B. Kostant and S. Sternberg, "Hamiltonian group actions and dynamical systems of Calogero type" Comm. Pure Appl. Math. 31 (1978), 481-508

[14] P.D. Lax, "Integrals of nonlinear equations of evolution and solitary waves", Comm. Pure Appl. Math. 21 (1968), 467-490

[15] C.M. Marle, "Symplectic manifolds, dynamical groups and Hamiltonian mechanics", in Differential geometry and relativity, M. Cohen and M. Flato eds., Reidel, Boston 1976

[16] G. Marmo, E.J. Saletan, A. Simoni and B. Vitale, Dynamical systems. A differential geometric approach to symmetry and reduction, Wiley, Chichester 1985

[17] A.I. Neishtadt, "On calculation of stability loss delay time for dynamical bifurcations", in: XIth International Congress of Mathematical Physics, D. Iagolnitzer ed., International Press, Cambridge (MA-USA) 1995

[18] S. Shnider and P. Winternitz, "Classification of systems of nonlinear ordinary differential equations with superposition principles", J. Math. Phys. 25 (1984), 3155-3165

[19] H. Thieme, "Convergence results and a Poincaré-Bendixson trichotomy for asymptotically autonomous differential equations", J. Math. Biol. 30 (1992), 755-763

[20] S. Walcher, "On differential equations in normal form", Math. Ann. 291 (1991), 293314

[21] J. Wei and E. Norman, "Lie algebraic solution of linear differential equations", $J$. Math. Phys. 4 (1963), 575-581; "On global representations of the solutions of linear differential equations as a product of exponentials", Proc. A.M.S. 15 (1964), 327-334

[22] P. Yanguas, "Lowering the dimension of polynomial vector fields in $R^{2}$ and $R^{3}$ ", Chaos 11 (2001), 306-318; J. Palacian, "Invariant manifolds of an autonomous ordinary differential equation from its generalized normal forms", Chaos 13 (2003), 1188-1204 